\title{
Análise do mercado de produtos orgânicos: estudo de caso de feira em Ponta Grossa, PR
}

\section{Analisys of the Market of Organic Products:A Case Study of Farmers Market in Ponta Grossa, Paraná State}

\author{
Ricardo SMOLINSKI* \\ Eziquiel GUERREIRO** \\ Augusta Pelinski RAIHER ${ }^{* * *}$
}

\begin{abstract}
RESUMO
O objetivo deste trabalho consiste em analisar o mercado de produtos orgânicos no Paraná, verificando o crescimento da sua produção em comparação aos produtos tradicionais, analisando a elasticidade-preço da demanda dos produtos orgânicos e identificando as principais características dos seus consumidores. Para tanto, utilizaram-se dados secundários e dados oriundos de uma pesquisa de mercado feita no município de Ponta Grossa, Paraná. Os resultados demonstraram que o mercado, tanto em termos de oferta de produtos orgânicos como em relação ao número de agricultores, está em crescimento. Na análise quanto ao comportamento da demanda por esses bens, principalmente no que se refere à sensibilidade dos consumidores frente aos seus preços, verificou-se que os produtos orgânicos são bens elásticos, onde a quantidade demandada responde fortemente às variações no preço. Mais precisamente, observou-se que as pessoas com maior renda estão dispostas a pagar mais por produtos orgânicos, do mesmo modo que pessoas que têm maior escolaridade também estão dispostas a pagar mais por esses bens, destacando que o principal motivo pelo qual se consomem orgânicos está na preocupação com a saúde.
\end{abstract}

Palavras-chave: agricultura orgânica; Paraná.

\begin{abstract}
The purpose of this study is to analyze the market of organic products in Paraná, checking the growth of their production in comparison to traditional products, analyzing the price elasticity of demand for organic products, and identifying the main characteristics of their consumers. To this end, we used secondary data and data from market research done in the city of Ponta Grossa, Paraná State. The results showed that the market both in terms of supply of organic products as to the number of farmers is growing. In analyzing the behavior of demand for these goods, especially with regard to sensitivity of consumers compared to

\footnotetext{
" Graduando em Economia pela Universidade Estadual de Ponta Grossa. Email: kadibr@yahoo.com.br

"* Mestre em Economia. Professor do Departamento de Economia da Universidade Estadual de Ponta Grossa - UEPG. Email: eziquiel@uepg.br

*** Doutora em economia. Professora adjunta do departamento de economia da Universidade Estadual de Ponta Grossa - UEPG. Email: apelinski@gmail.com
} 
their prices, we found that organic products are durable and elastic, as the demanded quantity responds strongly to price changes. More precisely, it was observed that people with higher income are willing to pay more for organic products in the same way that people who have higher education also are willing to pay more for these assets, highlighting that the main reason for consuming organic is their health concern.

Key-words: organic agriculture; Paraná State.

\section{Introdução}

A origem da agricultura moderna está ligada às descobertas do século XIX, a partir de estudos dos cientistas como Saussure, Boussingault e Liebig, os quais derrubaram teorias como a do húmus, argumentando acerca da capacidade das plantas obterem seu carbono a partir da matéria orgânica do solo (DE JESUS, 1985).

Liebig difundiu a ideia de que quanto mais produtos químicos são colocados na terra, maior tende a ser a quantidade de alimentos produzidos. Junto com Boussingault, que estudou a fixação de nitrogênio atmosférico pelas plantas leguminosas, Liebig é considerado o maior precursor da "agroquímica". As descobertas de todos esses cientistas marcaram o fim de um longo período da Antiguidade até o século XIX, no qual o conhecimento agronômico era essencialmente empírico. Essa nova fase seria caracterizada por um período de rápidos progressos científicos e tecnológicos (EHLERS, 1996).

No início do século XX, Louis Pasteur, Sergei Winogradsky e Martinus Beijerinck, precursores da microbiologia dos solos, dentre outros, contribuíram com fundamentos científicos por meio dos quais contrapuseram às teorias de Liebig, ao provarem a importância da matéria orgânica nos processos produtivos agrícolas (EHLERS, 1996).

Conforme Frade (2000), mesmo com o surgimento de comprovações científicas a respeito dos equívocos de Liebig, os impactos de suas descobertas haviam extrapolado o meio científico e ganhado força nos setores produtivo, industrial e agrícola, abrindo um amplo e promissor mercado: o de fertilizantes. Na medida em que certos componentes da produção agrícola passaram a ser produzidos pelo setor industrial, ampliaram-se as condições para o abandono dos sistemas de rotação de culturas e da integração da produção animal à vegetal, as quais passaram a ser realizadas separadamente. Tais fatos deram início a uma nova fase da história da agricultura, conhecida como "Segunda Revolução Agrícola". São também parte desse processo o desenvolvimento de motores de combustão interna e a seleção e produção de sementes, além de outros itens apropriados pelo setor industrial. Destaca-se que estas inovações foram responsáveis por sensíveis aumentos nos rendimentos das culturas no século XX.

Contudo, a partir dos anos 1960, esse modelo de agricultura começou a dar sinais de sua exaustão, seja pelos desmatamentos, pela diminuição da fertilidade do solo, pelas erosões, pela qualidade dos produtos, pela contaminação das águas, pelos resíduos de agrotóxicos, entre outros. Desta forma, começou-se a demandar, principalmente a partir dos anos 1980, uma agricultura mais ecológica, fomentando a agricultura orgânica (EHLERS, 1996).

Com essa consciência ecológica, nos anos de 1980 e 1990, a proposta de uma agricultura sustentável ganhou força, passando a ser defendida por produtores, consumidores e até mesmo pelos governos. Segundo Benevides (2010), os produtos orgânicos, por serem identificados como ecológicos, começaram a ser muito bem aceitos pelo mercado e as iniciativas de produção orgânica passaram a ser bem sucedidas.

De acordo com Glass (1998), o cultivo da agricultura orgânica requer cuidados especiais, uma vez que são produtos isentos de agrotóxicos, os adubos são naturais, necessitando, desta forma, de um número maior de mão de obra para poder observar e proteger as plantações contra as pragas.

Neste contexto, a agricultura familiar é a que mais se adapta às características da agricultura orgânica, essencialmente por apresentar, em geral, área de plantio pequena, por ser diversificada, autossustentável e, principalmente, por ter mão de obra disponível. Como no Paraná 86\% das propriedades rurais têm área inferior a 50 hectares, então a produção orgânica poderia ser uma alternativa para a agricultura familiar paranaense, não somente para geração de uma maior renda, mas, principalmente, visando propiciar condições de trabalho mais saudáveis para esses agricultores.

Entretanto, será que existe mercado suficiente para absorver uma maior produção de orgânicos? Este é o objetivo de estudo deste trabalho, ou seja, analisar o potencial 
de mercado dos produtos orgânicos no Paraná. Mais precisamente, busca-se verificar o crescimento da produção dos produtos orgânicos em comparação aos tradicionais no Paraná, a partir de 1996; analisar a elasticidade-preço da demanda dos produtos orgânicos e identificar as principais características dos consumidores de produtos orgânicos.

Para isso, esse trabalho está dividido em cinco seções, incluindo esta. $\mathrm{Na}$ segunda seção faz-se levantamento teórico acerca da agricultura orgânica. Na sequência, os elementos metodológicos são apresentados. Os resultados contemplam a quarta seção. Por fim, apresentam-se as considerações finais.

\section{Agricultura orgânica}

O homem primitivo sobrevivia com a ajuda da natureza, explorando a força natural da terra, cuidando das suas plantações, usando a própria natureza para adubar a terra. Entretanto, com a evolução tecnológica e química, passou-se a utilizar alguns recursos com o objetivo de melhorar a produção, sem muito pensar nas consequências dessas investidas. Buscava-se apenas proteger as plantações contra as pragas, aumentar a quantidade produzida, deixando em segundo plano a preocupação com a qualidade dos alimentos, com o meio ambiente e com a saúde.

A consequência foi que ao longo do tempo o meio ambiente foi devastado pelo homem, principalmente pelo uso incorreto dos recursos naturais. Além do uso de produtos químicos na agricultura, houve também extrações excessivas de madeiras, destruição da camada de ozônio, provocando mudanças climáticas, poluições hídricas e extinção de espécies (KHATOUNIAN, 2001).

O ser humano vem passando por diversas modificações no modo de pensar em relação aos recursos naturais. $\mathrm{Na}$ antiguidade, o homem sentia temor das forças da natureza. Segundo Khatounian (2001), enquanto se tinha uma baixa população o temor era pouco constatado; entretanto, após a evolução tecnológica, principalmente depois das bombas atômicas de Hiroshima e Nagasaki, a humanidade se sentiu mais dominante sobre tais forças.

Em meados de 1970, começaram a ser usados pacotes de insumos químicos: adubos, inseticidas, fungicidas e ainda uma infinidade de variedades modernas que, ao longo do processo, haviam sido selecionadas para bem aproveitar esses insumos. Foi onde a agricultura se tornou dependente da indústria química. Existia um esforço para convencer os produtores de que a agricultura, com a utilização de produtos químicos, era um meio eficiente de resolver problemas ligados à nutrição mineral e à sanidade vegetal.

Desta forma, a agricultura moderna passou a ser um verdadeiro processo industrial, os produtos que eram bens finais se transformaram em matérias-primas para as indústrias de alimentos. Foram deixados de lado alguns aspectos importantes, como a qualidade biológica e a sanidade dos alimentos (ausência de resíduos tóxicos), assim como a estabilidade das propriedades agrícolas e a conservação dos recursos naturais (AMBROSANO, 1999).

O modo de produção utilizando insumos químicos estendeu-se pelo mundo todo, resultando numa generalização de problemas causados por este modelo de cultivo, como também fez com que se desenvolvessem novos modos de produção, buscando o menor impacto ao meio ambiente.

Exemplo disso são os dados divulgados pela Sinitox (2008), demonstrando que em 2006 teve-se 15.783 casos de intoxicação por agrotóxicos no Brasil, dos quais 6.297 foram por produtos de uso agrícola, chegando a 186 óbitos registrados. Embora sejam altos esses dados, eles ainda não reportam a realidade dos fatos, uma vez que são dados vindos do Centro de Controle de Intoxicações, localizado em centros urbanos e que na grande maioria não cobre várias regiões produtoras da zona rural. Neste mesmo estudo, foi avaliado o nível de contaminação por agrotóxicos na zona rural, que teve como resultado uma variação de 3 a $23 \%$ (ALMEIDA; GARCIA, 1991; FARIA et al., 2000; GONZAGA; SANTOS, 1992).

Uma vez que para a produção de produtos orgânicos o uso de agrotóxicos é proibido, então a sua prática tende a reduzir o número de pessoas intoxicadas. Como a grande maioria dos produtores orgânicos trabalha com a família, em pequenas propriedades, passou-se a ter uma maior conscientização dos problemas que o agrotóxico traz, identificando um produto que é de melhor qualidade e sabor - o orgânico - e que também pode proporcionar um maior retorno financeiro.

A produção que resulta num impacto negativo menor ao meio ambiente ficou conhecida como agricultura alternativa ou orgânica, diferenciando-se do modelo da agricultura moderna. De acordo com Ambrosano (1999), o método alternativo de agricultura está inserido numa profunda mudança da sociedade frente aos recursos naturais, no contexto de defesa e proteção ambiental.

Os produtos orgânicos são obtidos em sistemas de produção onde não são utilizados fertilizantes sintéticos 
solúveis, agrotóxicos e transgênicos. Isso significa que são mais seguros para o consumo, além do que, as responsabilidades social e ambiental são características fundamentais na produção orgânica. Além disso, têm como base os princípios agroecológicos que contemplam o uso saudável e responsável do solo, da água, do ar e dos demais recursos naturais, de modo a reduzir as formas de contaminação e desperdício desses elementos, contribuindo para o desenvolvimento sustentável. As propriedades orgânicas também buscam diversificar e integrar a produção de espécies vegetais e animais com o objetivo de criar ecossistemas mais equilibrados e que ajudem a manter a biodiversidade (MAPA, 2009).

Segundo Darolt (2002), além de existir um conjunto de técnicas para serem seguidas pelos agricultores orgânicos, eles devem seguir uma ética orgânica, baseada em alguns princípios: o primeiro é identificar o solo como fonte e vida; o segundo princípio considera mais importante alimentar o solo do que a planta; o terceiro é a prevenção, a utilização de sistemas diversificados de produção, a independência do agricultor, o respeito à saúde e finalmente o respeito à natureza.

É importante frisar que o Brasil acompanhou o movimento mundial. Na década de 1970, houve um período de expansão no uso de agrotóxicos. Os poucos contrários ao padrão de produção agroquímico eram hostilizados e ridicularizados. Nas décadas de 1980 e 1990, as organizações ligadas com a agricultura orgânica cresceram. Os números de feiras que comercializavam esses produtos se multiplicaram, principalmente nas capitais. Além disso, o mercado atacadista também aderiu à comercialização de produtos orgânicos (KHATOUNIAN, 2001).

A agricultura orgânica no Brasil produz soja, açúcar mascavo, hortaliças, frutas, cereais, leguminosas e vários outros produtos, porém, a produção orgânica de animais ainda é muito pequena.

De acordo com Khatounian (2001), a agricultura orgânica está obtendo certo êxito, em que, mesmo sem o apoio necessário, se observa um maior desenvolvimento. No Paraná, a agricultura orgânica teve início na década de 1980, com os primeiros trabalhadores sendo organizados por produtores orgânicos na cidade de Agudos do Sul (DAROLT, 2002).

No princípio de 1986, houve uma iniciativa pioneira em Curitiba, a criação da chácara Verde Vida, na Colônia Faria, município de Colombo. Esta chácara iniciou sua produção baseada em princípios da agricultura biodinâmi- ca e começou sua produção com olerícolas (hortaliças) e pequenos animais, como aves e coelhos (DAROLT, 2002).

Atualmente, o Paraná se destaca na produção de orgânicos no país, praticada principalmente pela agricultura familiar. As principais culturas plantadas são a soja e a cana para açúcar mascavo, merecendo destaque também as culturas de hortaliças, frutas, arroz, feijão, milho, café, plantas medicinais, trigo e mandioca (HAMERSCHMIDT, 2006).

\section{Metodologia}

Os dados utilizados neste estudo foram coletados através de fontes como o MAPA (2010), Planeta Orgânico (2009), SEAB (2008) e Emater (2010). Além disso, fez-se uma pesquisa de mercado, via entrevistas, cujo objetivo central estava em determinar o quanto os consumidores estariam dispostos a pagar a mais pelos produtos orgânicos e quais as principais características desses consumidores.

\subsection{Coleta de dados: pesquisa de mercado}

Os dados foram coletados através de observações diretas, realizadas por meio de entrevistas, aplicadas a cem consumidores de verduras na feira de Ponta Grossa, no mês de junho de 2010. A entrevista constituiu-se das seguintes perguntas:

- Conhece produtos orgânicos?

- Consome produtos orgânicos?

- Grau de escolaridade:

- Renda familiar:

- O motivo do consumo de produtos orgânicos:

- Qual o percentual estaria disposto a pagar a mais pelo produto orgânico, em relação ao preço do produto não orgânico?

O cálculo do tamanho da amostra (n) foi efetuado por (3.1).

$$
\mathrm{n}=\frac{\mathrm{N}}{1+(\mathrm{N}-1) \mathrm{e}^{2}}
$$

Onde e é o erro máximo tolerado e $\mathbf{N}$ é a população (BERNI, 2002).

Foi constatado, por meio de pesquisa realizada diretamente com os feirantes, que o número médio de consumidores que frequentam a feira da cidade de Ponta Grossa, 
Paraná, é de 2.000 pessoas. Desta forma, considerando um erro máximo de $10 \%$ e uma população de 2.000 pessoas, o tamanho da amostra que foi utilizada correspondeu a 100 pessoas. Destaca-se que os consumidores foram escolhidos aleatoriamente, de modo a garantir a representatividade da amostra, ressaltando que os dados foram coletados em duas visitas à feira ${ }^{1}$.

\subsection{Elasticidade-preço da demanda}

A elasticidade de uma variável Y em relação a uma variável X é (3.2):

$$
E=\frac{\Delta \% Y}{\Delta \% X}=\frac{\frac{\Delta Y}{\frac{Y}{\Delta X}}}{X}=\frac{\Delta Y}{\Delta X} \cdot \frac{X}{Y}
$$

Em um modelo de regressão linear estimado: $\mathrm{Y}=\mathrm{a}$ + bX, a inclinação da reta é dada por

$$
b=\frac{\Delta Y}{\Delta X}
$$

Sendo assim, a elasticidade de $\mathrm{Y}$ em relação a $\mathrm{X}$ pode ser estimada por:

$$
E=b \cdot \frac{X_{i}}{Y_{i}}
$$

Como a elasticidade é diferente para cada ponto da reta de uma regressão, é usual determinar a elasticidade no ponto de médias $(\bar{X}$ e $\bar{Y})$, sendo um ponto representativo na reta de regressão (MATOS, 2001). Tem-se:

$$
E=b_{i} \cdot \frac{\bar{X}_{i}}{\bar{Y}_{i}}
$$

Considerando que o modelo de demanda em estudo é não linear $\left(Y_{i}=\beta_{0} X_{i}{ }^{\beta_{1}} \cdot e\right)$, então, para linearizá-lo, foram aplicados logaritmos nos dois lados da equação (3.6).

$$
\ln Y=\beta_{0}+\beta_{1} \ln X+\ln e
$$

Onde: $\ln \mathrm{Y}$ é o logaritmo da quantidade demanda e $\log \mathrm{X}_{\mathrm{o}}$ logaritmo da variação do preço.

Segundo MATOS (2001), estas transformações são feitas antes de se estimar o modelo de regressão linear, um modelo do tipo potência ou Log-log, destacando que, após transformado, fica (3.6).

Neste caso a elasticidade de $\mathrm{Y}$ em relação X é dada pelo próprio $\beta_{1}$.

\subsubsection{Modelo empírico}

Considerando a metodologia acerca da elasticidade-preço da demanda, utilizou-se, então, a estimação do modelo econométrico Log-log ou modelo de elasticidade constante, visando captar a sensibilidade dos consumidores frente às variações nos preços dos produtos orgânicos (modelo 3.7), estimativa feita pelo programa SAS (Statistical Analysis Software), pelo método dos Mínimos Quadrados Ordinários.

$$
D=f(L \bar{V} P)
$$

Onde: LD representa a demanda e $\mathbf{L P}$ o preço representado em valor porcentual acima do valor do produto não orgânico.

Os resultados foram avaliados pelos critérios teóricos, estatísticos e econométricos. Neste último, o modelo precisou passar pelos testes de normalidade residual e homocedasticidade. Para isso realizaram-se os testes: Shapiro-Wilk (o qual mensura se os resíduos apresentam distribuição normal) e Test of First and Second Moment Specification (diagnóstico de heterocedasticidade).

\subsection{Taxa geométrica de crescimento}

Para analisar a evolução da produção de orgânico versus a produção convencional, foi calculada a taxa geométrica de crescimento (TGC), utilizando-se o Método dos Mínimos Quadrados Ordinários em regressão linear.

Dada a função:

\footnotetext{
${ }^{1}$ A metodologia usada na coleta de informação de consumidores de produtos orgânicos em Ponta Grossa foi a mesma realizada por Cerveira e Castro (2010).
} 


$$
\mathrm{V}_{\mathrm{n}}=\mathrm{V}_{0}(1+\mathrm{r})^{\mathrm{T}}
$$

Onde: $\mathbf{V}_{\mathbf{n}}$ indicam os valores da variável em estudo no tempo $(\mathrm{T}=1,2, \ldots \mathrm{n})$; $\mathbf{r}$ é a taxa de crescimento; $\mathbf{V}_{\mathbf{0}}$ representa o valor da variável analisada num dado ano; e $\mathbf{V}_{\mathbf{0}} \mathbf{( 1}$ $+\mathbf{r}$ ) representa o crescimento dessa variável por unidade de tempo.

Aplicando logaritmo à expressão (3.8) tem-se:

$$
\mathrm{LnV}_{\mathrm{n}}=\ln \mathrm{V}_{0}+\mathrm{T} \cdot \ln (1+\mathrm{r})
$$

Tal expressão correspondente à equação:

$$
\mathrm{Y}_{\mathrm{i}}=\mathrm{a}+\mathrm{bX}
$$

Na qual: $Y_{i}=\ln V_{n} ; a=\ln V_{0} ; b=\ln (1+r)$ e $n$ é o período da variável que representa o tempo.

Tendo-se $b=\log (1+r), 1+r=\operatorname{antlog} b$ e $r=\operatorname{antlog}$ $\mathbf{b}-\mathbf{1}$, onde $\mathbf{r}$ corresponde à taxa média de crescimento do período que se analisa.

\subsection{Correlação de Spearman}

Com o objetivo de identificar quais os possíveis fatores que contribuem para que o consumidor esteja disposto a pagar a mais por um produto orgânico em relação ao preço do produto convencional, analisou-se a associação existente entre esta disposição, a renda e a escolaridade dos consumidores, através do cálculo da correlação de Spearman (3.11). Esse coeficiente, segundo Bisquera; Sarriera e Martinez (2004), capta a associação existente entre duas variáveis independentes se a associação existente é linear ou não. Destaca-se que os dados para fazer os cálculos acerca das correlações são oriundos da pesquisa que se fez, conforme foi exposto na seção 3.1 .

$$
r=1-\frac{6 \sum d_{i}^{2}}{n\left(n^{2}-1\right)}
$$

Onde: $\mathbf{r}=$ Coeficiente de correlação de Spearman; $\mathbf{d i}=$ Diferença de postos para par de observações; $\mathbf{n}=$ Número de pares.

\section{Análise dos resultados}

\subsection{Evolução da produção de orgânicos no Paraná}

No Gráfico 1, está evidenciado o percentual de agricultores orgânicos nas regiões do Brasil. Destaca-se que a região

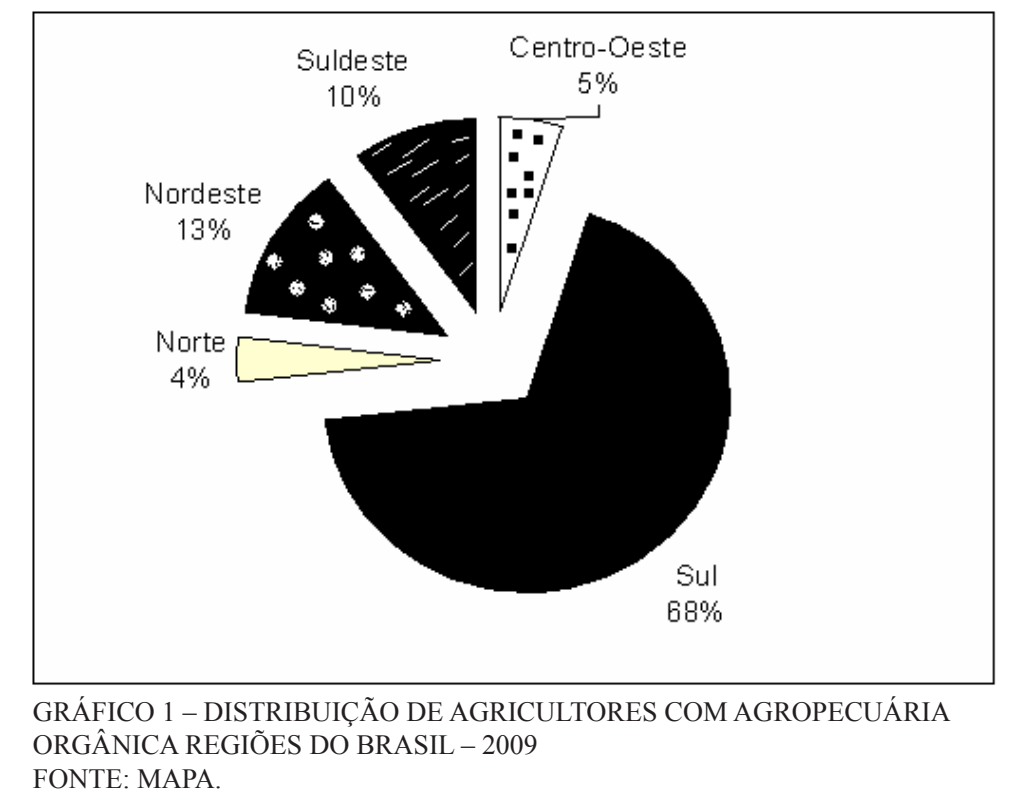


Sul é a que tem o maior percentual de produtores orgânicos. No caso do Paraná, a produção de orgânicos vem ganhando grande destaque, sendo um dos Estados com maior número de agricultores certificados (em torno de $25 \%$ do total).

A grande maioria da produção orgânica é realizada por pequenas propriedades de caráter familiar, produzindo uma grande diversidade de produtos, especialmente frutas e hortaliças (PLANETA ORGÂNICO, 2009). No Paraná, uma estimativa apresentada pela Agência de Notícias do Estado do Paraná (2009) revela que $86 \%$ das propriedades produtoras de orgânicos têm área inferior a 50 hectares.

Em relação ao número de produtores, de acordo com o Gráfico 2, visualiza-se que na safra 2008/2009 cerca de 4.750 agricultores orgânicos estavam atuando no Paraná. Em relação à safra anterior, observa-se uma pequena queda; entretanto, no decorrer dos anos teve-se uma significativa evolução, saindo de 450 agricultores orgânicos em

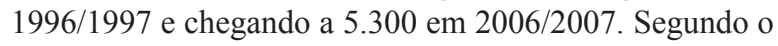
Planeta Orgânico (2009), essa grande inserção dos agricultores na produção de orgânicos é motivada principalmente pelo aumento na demanda por estes bens e pelo desenvolvimento de novos meios de comercialização.

Seab (2009) destaca que esta evolução positiva é decorrente do grande número de assentados rurais, das reservas indígenas e comunidades de quilombolas existentes, as quais seguem os preceitos da Agroecologia. Além disso, no Paraná essa atividade possui um alto nível de organização, existindo muitas ONGs que trabalham no setor, estando reunidas em um Fórum Estadual. Ao mesmo tempo, em 2007, instituiu-se a Câmara Setorial de Agricultura Orgânica do Paraná, envolvendo entidades governamentais e da sociedade civil organizada, com o objetivo comum de discutir e propor soluções aos problemas que dificultam o desenvolvimento da agricultura orgânica no Estado (SEAB, 2009).

Quanto aos fatos da expansão territorial e da topografia, o Paraná possui regiões com condições de clima e solo diferentes de outras regiões do Brasil e isto permite uma produção diversificada, vindo ao encontro das necessidades dos consumidores, existindo ainda a necessidade de um trabalho de logística, visando a uma maior integração entre as regiões.

De acordo com o Planeta Orgânico (2009), a produção de hortaliças no Paraná concentra-se em torno das grandes cidades, com destaque para Curitiba e Londrina, onde encontra-se a maior diversidade de produtos. Observam-se, também, polos de produção de açúcar mascavo nas regiões de Jacarezinho e Francisco Beltrão. A região Norte se sobressai na produção do café orgânico e frutas tropicais. Na região de Ponta Grossa, a produção fica por conta dos grãos (milho, soja e feijão).

Existe um grande crescimento na produção das culturas de trigo e milho, com tendências de se intensificar, acompanhando o plantio de soja. A tecnologia de produção orgânica preconiza a rotação de culturas. No caso específico

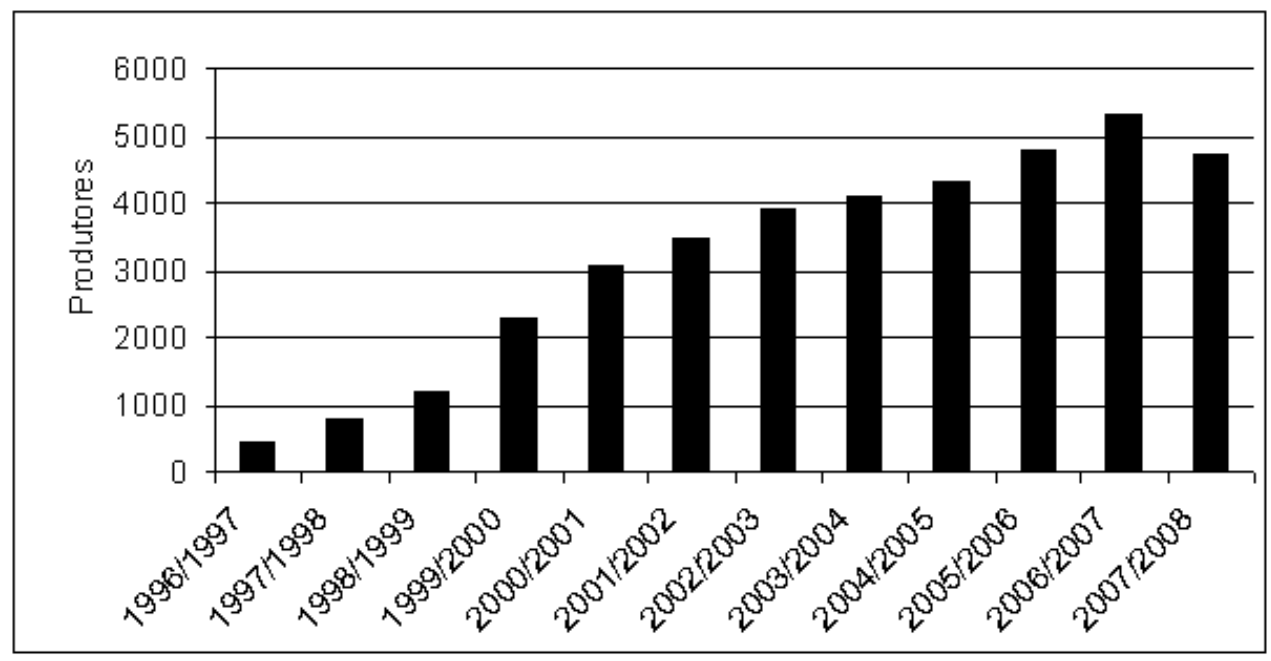

GRÁFICO 2 - NÚMERO DE PRODUTORES ORGÂNICOS NO ESTADO DO PARANÁ (1996-2008) FONTE: SEAB, 2009. 
do milho, a produção está sendo aumentada também devido ao crescimento da produção animal, a qual é importante para o equilíbrio do sistema orgânico pela produção de esterco, que, após o processo de compostagem, é usado para melhorar a fertilidade do solo.

Como mostra o Gráfico 3, no último levantamento de campo realizado pelos técnicos da Emater e do Deral, na safra de (2007/2008), mensurou-se uma produção equivalente a 124.323 toneladas de produtos orgânicos no Paraná. Houve um aumento de 15,9\% em relação à safra anterior, destacando-se a produção da soja em relação à área plantada. Em termos de produção, a cana-de-açúcar (destinada para o açúcar mascavo e para a cachaça) se sobressai, seguida pela mandioca e pelo grupo das hortaliças.

É importante destacar que, neste levantamento apresentado no Gráfico 3, considerou-se como produtores de orgânicos somente aqueles que já possuíam certificação ou que estavam em fase de conversão e ainda os que eram acompanhados por técnicos da EMATER ou de ONGs que atuam no setor. Neste último caso, os agricultores são avaliados pelo grau de comprometimento com os princípios da agroecologia. Assim, estima-se que $60 \%$ da produção total apresentada no Gráfico 3 seja de produto orgânico certificado, sendo o restante considerado orgânico em conversão e/ou não certificado.

No Paraná, $86 \%$ das propriedades rurais têm área inferior a 50 hectares, tornando-se importante o incentivo da produção de orgânicos para tentar obter maior rentabilidade por área destas propriedades. Neste sentido, olericultura, fruticultura, agricultura orgânica e ecoturismo, são opções melhores que o cultivo tradicional de grãos, onde se exige escala de produção.

Outro fator que incentiva a produção de orgânicos é a legislação ambiental, que também contribui a favor da agricultura orgânica. Em áreas de proteção ambiental ou em mananciais, é dificultada ou até mesmo proibida a prática de agricultura convencional.

Para que se tenha um crescimento constante, é preciso resolver alguns problemas inerentes à produção orgânica: primeiro, é necessário concluir a regulamentação da Lei 10.831 , de 23/12/2003, referente ao setor, para melhor ordenar e propiciar credibilidade; segundo, existem dificuldades com a assistência técnica, uma vez que a produção de orgânicos é complexa e exige conhecimentos, os quais muitas vezes são deficientes; e a carência de pesquisas para identificar como ocorrem as relações entre as diferentes espécies de uma comunidade e também métodos de controle de pragas e doenças.

A agricultura orgânica é uma atividade com grande potencial, com uma demanda crescente, uma vez que as pessoas estão cada vez mais se preocupando com a saúde e tendo consciência da necessidade de se proteger o meio ambiente.

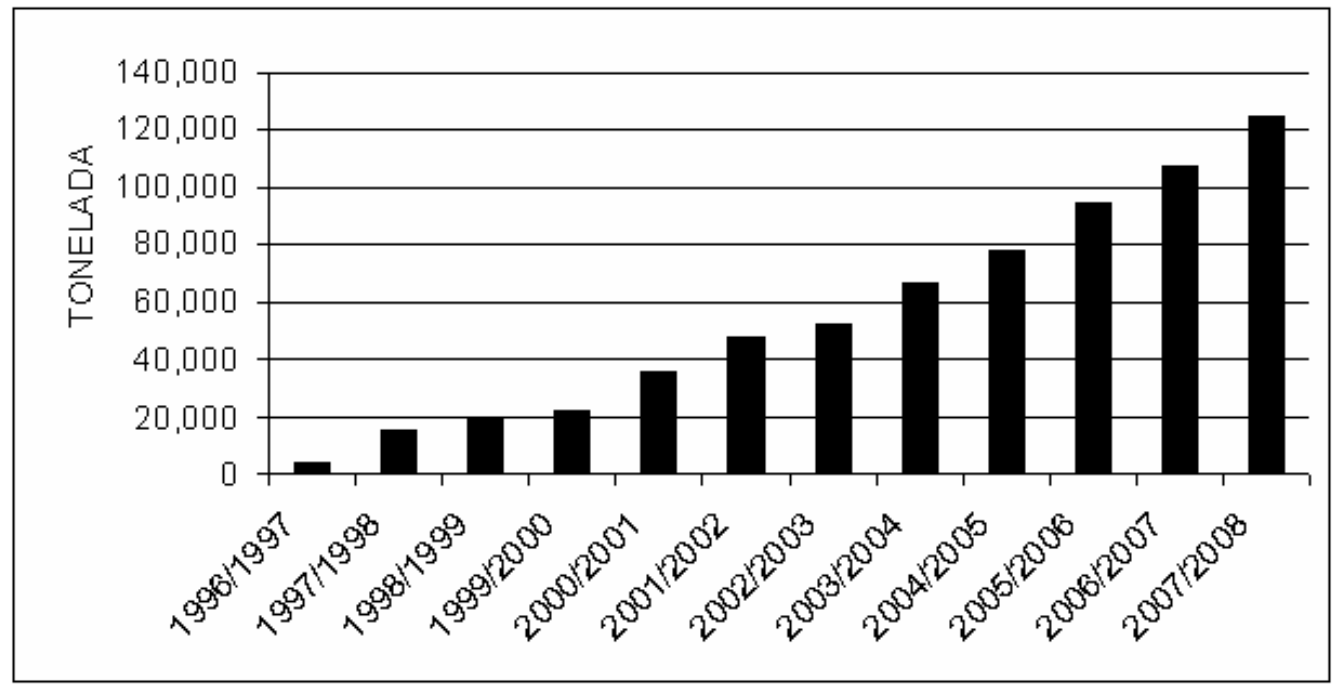

GRÁFICO 3 - VOLUME DE PRODUÇÃO ORGÂNICA NO ESTADO DO PARANÁ (1996-2008) FONTE: EMATER 


\subsubsection{Agricultura orgânica versus convencional no Paraná}

Na Tabela 1, apresenta-se a produção da agricultura convencional e a produção de orgânicos do Paraná. Nota-se que a quantidade da produção de orgânicos em relação à convencional é baixa; entretanto, o crescimento da agricultura orgânica em relação à convencional é alto, demonstrando um mercado em crescimento.

TABELA 1 - PRODUÇÃO AGRÍCOLA DO PARANÁ - 1996/1997 A 2009/2010 (EM T)

\begin{tabular}{c|c|c|c}
\hline Safra & $\begin{array}{c}\text { Produtos } \\
\text { Convencionais } \\
(\mathrm{A})\end{array}$ & $\begin{array}{c}\text { Produtos } \\
\text { Orgânicos } \\
(\mathrm{B})\end{array}$ & $\begin{array}{c}\text { Relação } \\
\text { B/A*100 } \\
(1)\end{array}$ \\
\hline $1996 / 1997$ & 17.362 .900 & 4.365 & 0,025139810 \\
$1997 / 1998$ & 17.097 .817 & 15.500 & 0,090654848 \\
$1998 / 1999$ & 18.795 .890 & 20.010 & 0,106459444 \\
$1999 / 2000$ & 15.672 .325 & 22.608 & 0,144254283 \\
$2000 / 2001$ & 23.977 .200 & 35.539 & 0,148219976 \\
$2001 / 2002$ & 21.553 .400 & 47.958 & 0,222507818 \\
$2002 / 2003$ & 29.044 .800 & 52.270 & 0,179963367 \\
$2003 / 2004$ & 25.714 .400 & 66.256 & 0,257661077 \\
$2004 / 2005$ & 22.474 .200 & 77.971 & 0,346935597 \\
$2005 / 2006$ & 25.344 .235 & 94.448 & 0,372660686 \\
$2006 / 2007$ & 28.400 .700 & 10.7230 & 0,377561116 \\
$2007 / 2008$ & 30.517 .600 & 124.323 & 0,407381314 \\
$2008 / 2009$ & $24.946 .400^{\mathrm{e}}$ & $124.785^{\mathrm{e}}$ & 0,500212456 \\
$2009 / 2010$ & $30.701 .842^{\mathrm{e}}$ & $135.412^{\mathrm{e}}$ & 0,441054970 \\
\hline TGC \% (1) & 5,26 & 36,65 & - \\
\hline FONTE: EMATER & $(2008), c 00(1)$ &
\end{tabular}

FONTE: EMATER (2008), com (1) calculado pela pesquisa.

Mais especificamente, observa-se, a partir dos dados da Tabela 1, que a produção convencional cresceu anualmente em torno de 5,26\%, enquanto que a produção orgânica se elevou num patamar igual a $36,65 \%$ ao ano, ou seja, um crescimento relativamente superior à agricultura convencional.

No Gráfico 4, a relação da produção dos dois sistemas (orgânico/convencional) também demonstra uma tendência de crescimento para os orgânicos, onde anualmente a produção vem ganhando um maior espaço.

Todos esses dados demonstram que a agricultura orgânica cresceu e tem uma tendência de crescimento futuro, em contraste com um crescimento mais modesto da agricultura convencional. Além disso, isso sinaliza que o mercado de orgânicos pode aumentar, sendo capaz de ofertar uma produção maior.

\subsection{Demanda dos produtos orgânicos}

\subsubsection{Influência do preço na demanda dos produtos orgânicos}

A partir da Equação 3.7, foi estimado o Modelo 4.1 para identificar a sensibilidade da demanda de produtos orgânicos (D) em relação ao preço pago pelo consumidor (P).

$$
\mathrm{LD}=\beta_{0}+\beta_{1} \mathrm{LP}+\mathrm{e}
$$

A seguir, são apresentadas as tabelas com os resultados das estimações, onde o coeficiente de determinação está representado por $\mathbf{R}^{2}$, a estatística de significância do modelo por $\mathbf{F}$, e ainda tem-se que: a representa nível de significância a 1\%, ${ }^{\text {b }}$ representa nível de significância a 5\%; $\mathrm{e}^{\mathrm{ns}}$ representa que o resultado foi não significativo até $5 \%$.

A partir dos resultados estimados através do Modelo 4.1, contidos na Tabela 2, pôde-se observar que o incremento de $1 \%$ no preço médio dos produtos orgânicos em relação aos preços médios dos produtos não orgânicos reduziria a demanda por produtos orgânicos em $4,64 \%$, ou seja, se os preços dos produtos orgânicos aumentarem em $21,55 \%$ acima dos preços dos produtos não orgânicos, a sua demanda tenderia a zero.

Ao contrário, uma pequena diminuição dos preços dos produtos orgânicos aumentaria mais que proporcional o seu consumo. Isso significa que os produtos orgânicos são bens elásticos, onde a quantidade demandada responde fortemente às variações no preço.

Alguns trabalhos, como por exemplo Pelinski e Guerreiro (2004), demonstram que alguns cultivos de orgânicos alcançam rentabilidade superior aos cultivos convencionais sem a necessidade de sobrevalorização de preços. Então, tem-se a possibilidade de se diminuir os preços dos orgânicos, mantendo ainda uma rentabilidade superior à produção convencional. E se essa diminuição ocorrer, considerando os resultados da Tabela 2, o efeito que se terá sobre a demanda será mais que proporcional. 


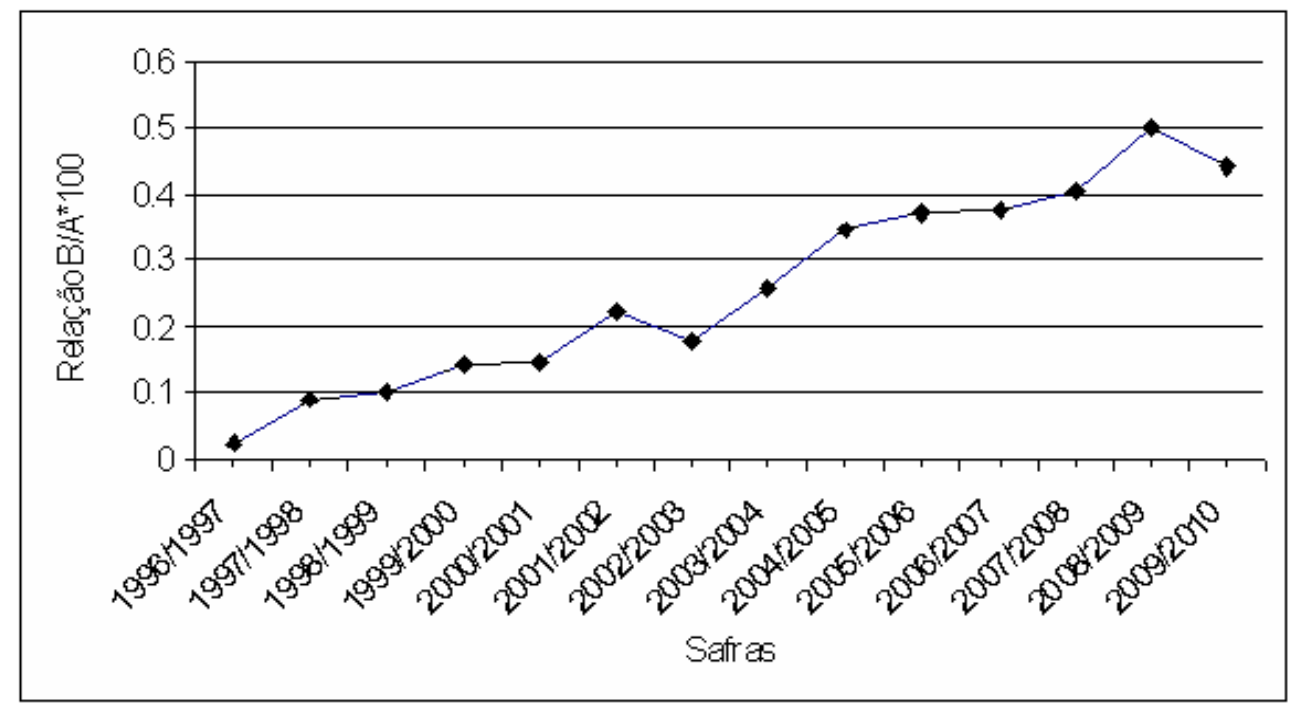

GRÁFICO 4 - PRODUÇÃO DA AGRICULTURA ORGÂNICA EM RELAÇÃO À CONVENCIONAL FONTE: Tabela 1.

TABELA 2 - RESULTADO DO MODELO ECONOMÉTRICO 4.1

\begin{tabular}{l|c|c|c|c}
\hline Modelo estimado & \multicolumn{2}{|c|}{ Parâmetros } & \multirow{3}{*}{} & \\
\cline { 2 - 4 } & Intercepto & $\begin{array}{c}\text { Coeficiente de } \\
\text { elasticidade }\end{array}$ & & \multirow{2}{*}{$\mathrm{R}^{2}$} \\
\cline { 2 - 5 } & $\mathrm{b}_{0}$ & $\mathrm{~b}_{1}$ & $\mathrm{~F}$ \\
\hline $\mathrm{LD}=\beta_{0}+\beta_{1} \mathrm{LVP}$ & $4,89^{\mathrm{a}}$ & $-4,64^{\mathrm{a}}$ & 93,42 & $85,14^{\mathrm{a}}$ \\
\hline
\end{tabular}

FONTE: dados da pesquisa.

NOTA: $a=$ significativo a $1 \%$, ns= não significativo até $5 \%$.

Para a avaliação de um modelo econométrico, alguns pressupostos devem ser satisfeitos para que tal estimativa seja válida em termos de confiabilidade. Um desses pressupostos é o da homocedasticidade, isto é, a variância dos resíduos obtidos seja constante, proporcionando uma distribuição uniforme ao longo da reta de regressão (MATOS, 2000). Para checar se o modelo atende ao pressuposto de variância constante, utilizou-se o Test of First and Second Moment Specification, o qual apresentou valor $p$ de 0,72 superior ao nível de significância adotado de 0,05 , o que permite aceitar a hipótese nula de ausência de heterocedasticidade, ou seja, o modelo é homocedástico (Tabela 3). Ao mesmo tempo, num modelo de regressão simples, as estimativas devem ser não tendenciosas e de variância mínima, isto é, eficientes, e para isso o modelo deve apresentar distribuição normal dos resíduos. Através da estatística de
Shapiro-Wilk $(0,94)$ apresentada na Tabela 3, constatou-se uma distribuição normal nos resíduos estatisticamente diferente de zero ao nível de significância adotado de 5\%. Portanto, aceitou-se a hipótese nula de presença de normalidade nos resíduos da regressão.

\section{TABELA 3 - TESTE DE HETEROCEDASTICIDADE E TESTE DE DISTRIBUIÇÃO NORMAL}

\begin{tabular}{c|c|c|c}
\hline \multicolumn{2}{c|}{$\begin{array}{c}\text { Test of First and Second } \\
\text { Moment Specification }\end{array}$} & \multicolumn{2}{c}{ Shapiro-Wilk } \\
\hline$\chi^{2}$ & $\operatorname{Pr}>$ ChiSq & W & $\operatorname{Pr}<\mathrm{W}$ \\
\hline $0,65^{\text {ns }}$ & 0,7230 & $0,94539^{\text {ns }}$ & 0,5858 \\
\hline
\end{tabular}

FONTE: Dados da pesquisa.

NOTA: - ChiSq $=$ qui-quadrado.

\subsubsection{Aspectos gerais da demanda por orgânicos}

Com o aumento crescente da demanda por produtos orgânicos no Paraná, está havendo uma maior conscientização quanto à diminuição no uso de produtos químicos prejudiciais à saúde dos agricultores e dos consumidores; maior preocupação com o meio ambiente e uma busca por um produto de melhor sabor. 
Alguns fatores que limitam o crescimento da demanda dos produtos orgânicos são: a oferta normalmente é inferior à demanda, tornando, desta forma, os preços dos produtos orgânicos muito acima dos preços dos produtos não orgânicos, desestimulando o seu consumo; falta de regularidade da oferta de produtos orgânicos durante o ano; pouca diversidade de alimentos orgânicos e poucas campanhas para esclarecimentos. Dentre estes, Darolt (2000) argumenta que o alto preço dos produtos orgânicos em relação aos não orgânicos é o principal fator desestimulador da demanda por tais produtos.

Assim, conforme foi observado na estimativa da Tabela 2, se houvesse uma redução nos preços dos produtos orgânicos, ter-se-ia um aumento na quantidade de venda bem acima da diminuição de preços, podendo aumentar significativamente os lucros dos produtores.

A Tabela 4 mostra que, das 100 pessoas entrevistadas, todas estão dispostas a pagar pelo menos $10 \%$ a mais pelos produtos orgânicos; $70 \%$ pagariam $20 \%$ a mais; com valor acima de $50 \%$, apenas $22 \%$ dos entrevistados estariam dispostos a pagar por produtos orgânicos, e apenas oito consumidores estariam dispostos a pagar um percentual de $100 \%$ a mais.

TABELA 4 - DEMANDA EM NÚMERO DE CONSUMIDORES POR NÍVEL DE PREÇO

\begin{tabular}{cc}
\hline Variação nos Preços $(\%)$ & Consumidores $\left(\mathrm{n}^{\mathrm{o}}\right)$ \\
\hline 1,10 & 100 \\
1,20 & 70 \\
1,30 & 42 \\
1,40 & 24 \\
1,50 & 22 \\
1,60 & 11 \\
1,70 & 9 \\
2,00 & 8 \\
\hline
\end{tabular}

FONTE: Dados da pesquisa.

Buscando identificar alguns fatores que possivelmente estão associados à disponibilidade dos consumidores em pagar mais por orgânicos em relação aos preços dos produtos convencionais, observou-se que a renda é um desses fatores. Conforme a Tabela 5 , nota-se que $42 \%$ dos entrevistados recebem mais que 10 salários mínimos e, considerando apenas esta classe, eles estariam dispostos a pagar 44,29\% a mais por produtos orgânicos em relação aos preços dos produtos convencionais (Apêndice 1). Já para as classes de até 2 salários mínimos e de 2 a 4, estariam dispostos a pagar pelo menos $10 \%$ a mais, uma vez que na entrevista havia a opção de não pagar nada a mais, isto é, só consumir pelo mesmo valor dos produtos convencionais.

Mais precisamente, a correlação apresentada na Tabela 6 demonstra existir uma associação positiva entre o nível de renda e a disposição para pagar a mais por produtos orgânicos, em que um aumento de renda significa uma maior disposição em pagar mais caro por alimentos orgânicos (e vice-versa). Esse resultado demonstra que o nível de renda interfere nas decisões das pessoas ao optar por uma melhor qualidade de produtos, sem agrotóxicos.

TABELA 5 - NÚMERO ABSOLUTO E RELATIVO DE CONSUMIDORES POR CLASSE DE RENDA

\begin{tabular}{c|cc}
\hline Classes de renda & \multicolumn{2}{|c}{ Consumidores } \\
\hline (SM) & $\left(\mathrm{n}^{\circ}\right)$ & $(\%)$ \\
Até 2 & 3 & 10,00 \\
De 2 a 4 & 11 & 10,00 \\
De 4 a 6 & 14 & 14,29 \\
De 6 a 8 & 11 & 19,09 \\
De 8 a 10 & 19 & 32,11 \\
$(+)$ de 10 & 42 & 44,29 \\
\hline
\end{tabular}

FONTE: Dados da pesquisa

NOTA: $\mathrm{SM}=$ salário mínimo

TABELA 6 - CORRELAÇÃO DE SPEARMAN ENTRE VARIÁVEIS SELECIONADAS

\begin{tabular}{l|c|c|c}
\hline Variáveis & Pagar a Mais & Escolaridade & Renda \\
Pagar a Mais & 1 & $0,50^{*}$ & $0,74^{*}$ \\
Escolaridade & $0,50^{*}$ & 1 & $0,50^{*}$ \\
Renda & $0,74^{*}$ & $0,50^{*}$ & 1 \\
\hline
\end{tabular}

FONTE: Resultado da Pesquisa. NOTA: * significativo a $1 \%$

Na Tabela 7, pode-se verificar que 59\% dos entrevistados têm nível superior completo e aceitam pagar 38,14\% a mais pelos produtos orgânicos; em contrapartida, 10\% dos entrevistados responderam que seu grau de instrução é de $1^{\circ}$ ou $2^{\circ}$ grau incompleto e estariam dispostos a pagar no máximo $10 \%$ a mais na substituição de produtos convencionais por produtos orgânicos (Apêndice 1). Ou seja, existe uma tendência de que quanto maior é a escolaridade das pessoas, maior é a sua disponibilidade em pagar a mais 
por produtos orgânicos. Essa associação é comprovada por meio da Tabela 6, onde se identifica essa correlação positiva entre essas variáveis.

Diante disso, pode-se considerar que aquelas pessoas que têm um maior grau de instrução possivelmente têm um acesso maior às informações com respeito aos benefícios que o consumo de orgânicos venha a trazer para a sua saúde, para o meio ambiente, entre outros, se disponibilizando, portanto, a pagar mais caro por esses alimentos. Ou, como na Tabela 6 consegue-se visualizar uma correlação entre renda e escolaridade, então esta maior disponibilidade dos consumidores com maior escolaridade em pagar a mais por orgânicos pode ser oriunda de uma maior renda que estes venham a ter.

TABELA 7 - NÚMERO ABSOLUTO E RELATIVO DE CONSUMIDORES POR GRAU DE ESCOLARIDADE

\begin{tabular}{c|c|c}
\hline \multirow{2}{*}{$\begin{array}{c}\text { Escolaridade } \\
\text { (grau de instrução) }\end{array}$} & \multicolumn{2}{|c}{ Consumidores } \\
\cline { 2 - 3 }$\left(\mathrm{n}^{\mathrm{o}}\right)$ & $(\%)$ \\
\hline $1^{\circ}$ grau & 7 & 10,00 \\
$2^{\circ}$ grau incompleto & 3 & 10,00 \\
$2^{\circ}$ grau & 17 & 22,94 \\
Superior Incompleto & 14 & 20,00 \\
Superior & 59 & 38,14 \\
\hline
\end{tabular}

FONTE: Dados da pesquisa

Outra pergunta realizada foi o motivo pelo qual o entrevistado substituiria o produto convencional pelo produto orgânico. Como resultado, constatou-se que todos têm como preocupação central a saúde, destacando que apenas $11 \%$ consomem orgânicos visando contribuir com o meio ambiente e não se teve citação para sabor e preço ou outros aspectos (Apêndice 1). Estes 11\% que citaram o meio ambiente pertencem ao grupo de escolaridade de nível superior, podendo-se chegar à conclusão da falta de informação a respeito de todos os benefícios dos produtos orgânicos para a sociedade, corroborando com a afirmação feita anteriormente, referente à maior disponibilidade das pessoas que possuem um maior grau de instrução em pagar mais caro por esses produtos, principalmente por ter um acesso maior às informações sobre os benefícios desses produtos.

\section{Considerações finais}

Os alimentos orgânicos são obtidos em sistemas de produção onde não são utilizados fertilizantes sintéticos solúveis, agrotóxicos e transgênicos. Isso significa que são mais seguros para o consumo. Ao mesmo tempo, dadas essas características, a sua produção requer cuidados especiais, necessitando de um número maior de mão de obra para poder observar e proteger as plantações contra as pragas. E é por isso que a agricultura familiar é a que mais se adapta à agricultura orgânica, essencialmente por apresentar, em geral, área de plantio pequena, por ser, na maioria das vezes, diversificada, autossustentável e, principalmente, por ter mão de obra disponível.

Como no Paraná a grande maioria das propriedades rurais tem área inferior a 50 hectares $(86 \%)$, então a produção orgânica poderia ser uma alternativa para a agricultura familiar, não somente para geração de uma maior renda, mas, principalmente, visando propiciar condições de trabalho mais saudáveis para esses agricultores.

Os resultados demonstraram que o mercado, em termos de oferta de produtos orgânicos no Paraná, está em franco crescimento, com uma taxa geométrica em torno de $36 \%$ ao ano contra cerca de $5 \%$ dos produtos não orgânicos. Esse crescimento também é comprovado por meio do número de agricultores envolvidos neste sistema de produção,

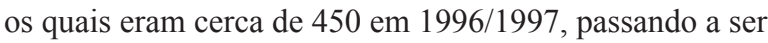
5.300 na safra 2006/2007. Alguns autores argumentam que esse crescimento da oferta é uma consequência direta do aumento da demanda por esses bens e é um resultado do desenvolvimento de novos meios de comercialização.

Ao se analisar o comportamento da demanda por esses bens, principalmente no que se refere à sensibilidade dos consumidores frente aos seus preços, os resultados demonstraram que os produtos orgânicos são bens elásticos, ou seja, a quantidade demandada responde fortemente às variações no preço. Mais precisamente, uma diminuição de $1 \%$ no preço médio dos produtos orgânicos em relação ao preço dos não orgânicos elevaria a sua demanda em $4,65 \%$. Como ainda é grande a diferença de preço entre os produtos orgânicos e os convencionais, então uma pequena queda dos preços dos primeiros elevaria significativamente seu consumo.

Entre os fatores que possivelmente estariam correlacionados com essa sensibilidade, observou-se que as pessoas com maior renda estão dispostas a pagar mais por produtos orgânicos, do mesmo modo que aquelas com 
maior escolaridade também estão dispostas a pagar mais por esses bens.

Dentre os motivos pelos quais se demandariam orgânicos está, de forma unânime, a preocupação com a saúde $(100 \%$ dos entrevistados), vindo, na sequência, a preocupação com o meio ambiente (apenas 11\%).

Diante desses resultados, políticas públicas visando fomentar esse setor poderiam ser desenvolvidas, principalmente ações (como subsídios, treinamentos, assessoria

\section{Referências}

AGÊNCIA DE NOTÍCIAS DO ESTADO DO PARANÁ, 2009. Agricultura: Expansão da agricultura orgânica no Paraná. Disponível em: <http// www.aen.pr.gov.br>. Acesso em: maio 2010.

ALMEIDA, W. F.; GARCIA, E. G. Exposição dos trabalhadores rurais aos agrotóxicos no Brasil. Revista Brasileira de Saúde Ocupacional, v. 19, n. 72, p. 7-11, 1991.

AMBROSANO, E. Agricultura Ecológica. São Paulo: Livraria e Editora Agropecuária, 1999.

BENEVIDES, L. Como funciona a agricultura orgânica. Disponível em: $<$ http://ambiente.hsw.uol.com.br/agricultura-orgânica2.htm>. Acesso em: set. 2010.

BÊRNI, Duilio A. Técnicas de pesquisa em economia: transformando ideias em conhecimento. São Paulo: Saraiva, 2002.

BISQUERRA, R.; SARRIERA, J. C.; MARTINEZ, F. Introdução à estatística: um enfoque informático com o pacote estatístico SPSS. Porto Alegre: Artmed, 2001.

CERVEIRA, R.; CASTRO, M. C. Perfil socioeconômico dos consumidores de produtos orgânicos da cidade de São Paulo. Disponível em: <http://www.megaagro.com.br/organica/perfil_um.asp>. Acesso em: dez. 2010.

DAROLT, Moacir R. Agricultura orgânica: inventando o futuro. Londrina: IAPAR, 2002.

DE JESUS, E. L. Histórico e filosofia da agricultura alternativa. Revista Proposta, Rio de Janeiro: PTA-FASE, p. 34-40, 1985.

EHLERS, Eduardo. Agricultura sustentável: origens e perspectivas de um novo paradigma. São Paulo: Livros da Terra, 1996.

EMATER. Disponível em: $<$ http://www.emater.pr.gov.br/emater.php? emater $=2 \& \mathrm{mid}=87>$. Acesso em: dez. 2010. técnica) que resultassem numa diminuição dos preços dos alimentos orgânicos, tornando-o muito mais competitivo.

Outra frente de ação que poderia ser efetuada para fomentar esse sistema de produção seria a divulgação de informações para a população, esclarecendo o que realmente são os produtos orgânicos, seus benefícios para a saúde e para o meio ambiente, com o objetivo que todas as pessoas, independente do nível de instrução e classe de renda, tenham conhecimento sobre as vantagens desse produto.

FARIA, N.M.; FACCHINI, L.A; FASSA, A.G; TOMASI, E. Estudo transversal sobre saúde mental de agricultores da Serra Gaúcha (Brasil). Rev Saúde Pública, n. 33, p. 391-400, 1999.

FRADE, Carmem Oliveira. A construção de um espaço para pensar e praticar a Agroecologia na UFRRJ e seus arredores. Dissertação (Mestrado em Agroecologia) - CPDA/UFRRJ. Rio de Janeiro, 2000.

GLASS, D. J. The 1998 United status market for phytoremediatino. Needhamm: D. Glass Associates, 1998.

GONZAGA M. C.; SANTOS, S. O. Avaliação das condições de trabalho inerentes ao uso de agrotóxicos nos municípios de Fátima do Sul, Glória de Dourados e Vicentina - Mato Grosso do Sul. Revista Brasileira de Saúde Operacional, n. 20, p. 42-46, 1990.

HAMERSCHMIDT, I. Agricultura orgânica e segurança alimentar. Disponível em: $<$ http://www.planetaorganico.com. br/Iniberto.htm>. Acesso em: 01/12/2009.

KHATOUNIAN, C. A. A reconstrução ecológica da agricultura. Rio de Janeiro: Editora IAPAR, 2001.

MAPA. Artigos de politica agrícola. Disponível em: $<\mathrm{http}: / /$ www.agricultura.gov.br/>. Acesso em: 29/11/2009.

MATOS, O. C. de. Econometria básica: teoria e aplicações. 3. ed. São Paulo: Atlas, 2000.

PELINSKI, A; GUERREIRO, E. Os benefícios da agricultura orgânica em relação à convencional: ênfase em produtos selecionados. Publication/UEPG, 2004.

PLANETA ORGÂNICO. A sustentabilidade do sistema de agricultura orgânica. Disponível em: <http://www.planetaorganico.com.br/trabdarolt2.htm>. Acesso em: $01 \mathrm{dez} 2009$.

SECRETARIA DA AGRICULTURA E DO ABASTECIMENTO DO PARANÁ (SEAB). Agricultura Orgânica, 
2008. Disponível em: <http://www.seab.pr.gov.br>. Acesso em: dez. 2009.

SISTEMA NACIONAL DE INFORMAÇÕES TÓXICOFARMACOLÓGICAS (SINITOX). 2008. Disponível em:
$<$ http://www.fiocruz.br/sinitox_novo/cgi/cgilua.exe/sys/start. $\mathrm{htm}>$. Acesso em: dez 2009.

APÊNDICE 1 - TABELA DA PESQUISA REALIZADA EM CAMPO

\begin{tabular}{|c|c|c|c|c|c|}
\hline \multicolumn{6}{|c|}{ Dados da pesquisa de campo } \\
\hline Conhece & Consome & Motivo & Escolaridade & Renda & Percentual \\
\hline SIM & NAO & 1 & $2^{\circ}$ grau incompleto & de 2 a 4 & 10 \\
\hline SIM & NAO & 1 & $1^{\circ}$ grau & De 2 a 4 & 10 \\
\hline SIM & NAO & 1 & superior & De 4 a 6 & 10 \\
\hline SIM & NÃO & 1 & $1^{\circ}$ grau & até 2 & 10 \\
\hline SIM & NÃO & 1 & $1^{\circ}$ grau & até 2 & 10 \\
\hline SIM & NÃO & 1 & $1^{\circ}$ grau & até 2 & 10 \\
\hline SIM & NÃO & 1 & $1^{\circ}$ grau & De 2 a 4 & 10 \\
\hline SIM & NÃO & 1 & $2^{\circ}$ grau & De 4 a 6 & 10 \\
\hline SIM & SIM & 1 & superior incompleto & De 2 a 4 & 10 \\
\hline SIM & SIM & 1 & superior & De 2 a 4 & 10 \\
\hline SIM & NÃO & 1 & $1^{\circ}$ grau & De 2 a 4 & 10 \\
\hline SIM & SIM & 1 & superior incompleto & De 2 a 4 & 10 \\
\hline SIM & NÃO & 1 & $2^{\circ}$ grau incompleto & De 2 a 4 & 10 \\
\hline SIM & SIM & 1 & superior & De 2 a 4 & 10 \\
\hline SIM & NÃO & 1 & $2^{\circ}$ grau incompleto & De 2 a 4 & 10 \\
\hline SIM & NÃO & 1 & $1^{\circ}$ grau & De 2 a 4 & 10 \\
\hline SIM & NAO & 1 & $2^{\circ}$ grau & De 4 a 6 & 10 \\
\hline SIM & SIM & 1 & superior incompleto & De 4 a 6 & 10 \\
\hline SIM & NAO & 1 & $2^{\circ}$ grau & De 4 a 6 & 10 \\
\hline SIM & NAO & 1 & $2^{\circ}$ grau & De 4 a 6 & 10 \\
\hline SIM & SIM & 1 & superior incompleto & De 4 a 6 & 10 \\
\hline SIM & NÃO & 1 & $2^{\circ}$ grau & De 4 a 6 & 10 \\
\hline SIM & NÃO & 1 & $2^{\circ}$ grau & De 4 a 6 & 10 \\
\hline SIM & SIM & 1 & superior incompleto & De 4 a 6 & 10 \\
\hline SIM & NÃO & 1 & $2^{\circ}$ grau & De 4 a 6 & 10 \\
\hline SIM & NÃO & 1 & superior & De 6 a 8 & 10 \\
\hline SIM & NÃO & 1 & superior & De 6 a 8 & 10 \\
\hline SIM & SIM & 1 & $2^{\circ}$ grau & De 8 a 10 & 10 \\
\hline SIM & NÃO & 1 & superior & De 8 a 10 & 10 \\
\hline SIM & SIM & 1 & superior & $(+)$ de 10 & 10 \\
\hline SIM & SIM & 1 & superior & de 4 a 6 & 20 \\
\hline
\end{tabular}




\begin{tabular}{|c|c|c|c|c|c|}
\hline SIM & SIM & 1 & superior incompleto & de 4 a 6 & 20 \\
\hline SIM & SIM & 1 & superior & de 6 a 8 & 20 \\
\hline SIM & SIM & 1 & superior & de 6 a 8 & 20 \\
\hline SIM & NAO & 1 & superior & de 6 a 8 & 20 \\
\hline SIM & SIM & 1 & $2^{\circ}$ grau & de 6 a 8 & 20 \\
\hline SIM & SIM & 1 & superior & de 6 a 8 & 20 \\
\hline SIM & SIM & 1 & superior & de 6 a 8 & 20 \\
\hline SIM & NÃO & 1 & $2^{\circ} \mathrm{grau}$ & de 6 a 8 & 20 \\
\hline SIM & NÃO & 1 & superior & de 6 a 8 & 20 \\
\hline SIM & SIM & 1 & superior & De 8 a 10 & 20 \\
\hline SIM & NAO & 1 & superior & De 8 a 10 & 20 \\
\hline SIM & SIM & 1 & superior & De 8 a 10 & 20 \\
\hline SIM & NAO & 1 & superior incompleto & De 8 a 10 & 20 \\
\hline SIM & SIM & 1 & superior & De 8 a 10 & 20 \\
\hline SIM & NÃO & 1 & $2^{\circ} \mathrm{grau}$ & De 8 a 10 & 20 \\
\hline SIM & SIM & 1 & superior incompleto & De 8 a 10 & 20 \\
\hline SIM & NÃO & 1 & superior & De 8 a 10 & 20 \\
\hline SIM & SIM & 1 e 2 & superior incompleto & $(+)$ de 10 & 20 \\
\hline SIM & $\mathrm{NAO}$ & 1 & superior & $(+)$ de 10 & 20 \\
\hline SIM & SIM & 1 e 2 & superior & $(+)$ de 10 & 20 \\
\hline SIM & SIM & 1 & superior & $(+)$ de 10 & 20 \\
\hline SIM & SIM & 1 & superior & $(+)$ de 10 & 20 \\
\hline SIM & SIM & 1 & superior incompleto & $(+)$ de 10 & 20 \\
\hline SIM & SIM & 1 & superior incompleto & $(+)$ de 10 & 20 \\
\hline SIM & NÃO & 1 & superior & $(+)$ de 10 & 20 \\
\hline SIM & SIM & 1 & superior & $(+)$ de 10 & 20 \\
\hline SIM & NÃO & 1 & $2^{\circ}$ grau & $(+)$ de 10 & 20 \\
\hline SIM & SIM & 1 & superior & de 6 a 8 & 30 \\
\hline SIM & $\mathrm{NAO}$ & 1 & superior & De 8 a 10 & 30 \\
\hline SIM & SIM & 1 & superior & De 8 a 10 & 30 \\
\hline SIM & SIM & 1 & superior & De 8 a 10 & 30 \\
\hline SIM & SIM & 1 & superior & De 8 a 10 & 30 \\
\hline SIM & SIM & 1 & superior & De 8 a 10 & 30 \\
\hline SIM & SIM & 1 & superior & De 8 a 10 & 30 \\
\hline SIM & SIM & 1 & superior & $(+)$ de 10 & 30 \\
\hline SIM & SIM & 1 & superior & $(+)$ de 10 & 30 \\
\hline SIM & SIM & 1 & superior & $(+)$ de 10 & 30 \\
\hline SIM & SIM & 1 & superior & $(+)$ de 10 & 30 \\
\hline
\end{tabular}


SMOLINSKI, R. et al. Análise do mercado de produtos orgânicos: estudo de caso de feira...

\begin{tabular}{|c|c|c|c|c|c|}
\hline SIM & SIM & 1 & superior & $(+)$ de 10 & 30 \\
\hline SIM & SIM & 1 & 2 grau & $(+)$ de 10 & 30 \\
\hline SIM & SIM & 1 & superior & $(+)$ de 10 & 30 \\
\hline SIM & SIM & 1 & superior & $(+)$ de 10 & 30 \\
\hline SIM & SIM & 1 & superior incompleto & $(+)$ de 10 & 30 \\
\hline SIM & SIM & 1 & superior & $(+)$ de 10 & 30 \\
\hline SIM & SIM & 1 & superior incompleto & $(+)$ de 10 & 30 \\
\hline SIM & NAO & 1 e 2 & superior & $(+)$ de 10 & 40 \\
\hline SIM & SIM & 1 & superior & $(+)$ de 10 & 40 \\
\hline SIM & SIM & 1 & $2^{\circ}$ grau & de 4 a 6 & 50 \\
\hline SIM & SIM & 1 & superior & De 8 a 10 & 50 \\
\hline SIM & SIM & 1 & $2^{\circ}$ grau & $(+)$ de 10 & 50 \\
\hline SIM & SIM & 1 & superior incompleto & $(+)$ de 10 & 50 \\
\hline SIM & SIM & 1 & superior & $(+)$ de 10 & 50 \\
\hline SIM & SIM & 1 & $2^{\circ}$ grau & $(+)$ de 10 & 50 \\
\hline SIM & SIM & 1 e 2 & superior & $(+)$ de 10 & 50 \\
\hline SIM & SIM & 1 & $2^{\circ}$ grau & $(+)$ de 10 & 50 \\
\hline SIM & SIM & 1 & superior & $(+)$ de 10 & 50 \\
\hline SIM & SIM & 1 & superior & $(+)$ de 10 & 50 \\
\hline SIM & SIM & 1 & superior & $(+)$ de 10 & 50 \\
\hline SIM & SIM & 1 & superior & $(+)$ de 10 & 60 \\
\hline SIM & SIM & 1 e 2 & superior & $(+)$ de 10 & 60 \\
\hline SIM & SIM & 1 e 2 & superior & $(+)$ de 10 & 70 \\
\hline SIM & SIM & 1 & superior & De 8 a 10 & 100 \\
\hline SIM & SIM & 1 & superior & De 8 a 10 & 100 \\
\hline SIM & SIM & 1 & superior & $(+)$ de 10 & 100 \\
\hline SIM & SIM & 1 e 2 & superior & $(+)$ de 10 & 100 \\
\hline SIM & SIM & 1 e 2 & superior & $(+)$ de 10 & 100 \\
\hline SIM & SIM & 1 e 2 & superior & $(+)$ de 10 & 100 \\
\hline SIM & SIM & 1 e 2 & superior & $(+)$ de 10 & 100 \\
\hline SIM & SIM & 1 e 2 & superior & $(+)$ de 10 & 100 \\
\hline
\end{tabular}

FONTE: Dados da Pesquisa

NOTA: 1 representa saúde, 2 meio ambiente, 3 sabor e 4 outros.

Recebido em março de 2011.

Aceito em maio de 2011.

Publicado em junho de 2011. 